Abanico Veterinario. Enero-Diciembre 2021; 11:1-10. http://dx.doi.org/10.21929/abavet2021.14

Artículo Original. Recibido: 27/06/2020. Aceptado: 02/03/2021. Publicado: 25/03/2021. Clave: e2020-65.

\title{
Efecto de un protocolo de prostaglandina a corto plazo sobre la sincronización y resultados reproductivos en las cabras cíclicas
}

\author{
Effect of a short-term prostaglandin protocol upon synchronization and \\ reproductive outcomes in cyclic goats
}

\section{Rivas-Muñoz Raymundo 1 ID , Zúñiga-García Santiago ${ }^{2}$ ID , Arellano-Rodríguez Gerardo $^{3 \text { ID }}$, Arellano-Rodríguez Fernando ${ }^{3 \mathrm{ID}}$, Gaytán-Alemán Leticia ${ }^{3 \mathrm{ID}}$, Contreras- Villarreal Viridiana ${ }^{3^{*}}$ ID}

${ }^{1}$ Instituto Tecnológico de Torreón, Carretera Torreón-San Pedro de las Colonias KM 7.5, Ejido Ana, 27170, Torreón, Coahuila, México. 'Universidad Juárez del Estado de Durango, Facultad de Agricultura y Zootecnia, Venecia, Villanápoles, 35018, Gómez Palacio, Durango. México. ${ }^{3}$ Universidad Autónoma Agraria Antonio Narro, Unidad Laguna, Periférico Raúl López Sánchez, Valle Verde, 27054, Torreón, Coahuila, México. *Autor de correspondencia. Correo-e: dra.viridianac@gmail.com, raymundorivasmuoz@yahoo.com.mx, s_zuniga83@hotmail.com, gveterinarioarellano@gmail.com, fernandoarellano_13@yahoo.com.mx,zukygay_7@hotmail.com

\section{RESUMEN}

El objetivo del presente estudio fue evaluar la respuesta reproductiva de cabras cíclicas del norte de México $\left(26^{\circ} \mathrm{N}\right)$ sometidas a dos protocolos de administración de prostaglandinas (PG; 5 días vs 10 días) con el fin de sincronizar la respuesta estral. Se formaron 2 grupos de hembras caprinas adultas cíclicas de la raza Alpino francés. Al Grupo G5 $(n=10)$, se les administró la primera inyección de PG $(0.2 \mathrm{ml} ; 160 \mu \mathrm{g}$ cloprostenol) el d -5 y el grupo $\mathrm{G} 10(\mathrm{n}=10)$ recibió la primera inyección de PG el d -10 . Ambos grupos recibieron la segunda inyección de $P G$ el d 0 (noviembre 1). La respuesta reproductiva general después de la segunda inyección de $P G$ fue similar $(P>0.05)$ entre ambos tratamientos. Respuesta estral $=60 \%$ para ambos grupos, latencia al estro $=68 \mathrm{~h}$ vs $52 \mathrm{~h}$, ovulación $=80 \%$ vs $60 \%$ (G5 vs G10, respectivamente; $P>0.05)$. Debido a que no hubo diferencias entre grupos, la administración del tratamiento G5 parece ser una alternativa interesante para la utilización de un protocolo corto ya que generó una respuesta reproductiva importante.

Palabras clave: Cabras Alpino Francés, época reproductiva, sincronización estral, prostaglandinas, respuesta reproductiva.

\section{ABSTRACT}

The aim of the study was to evaluate the reproductive response of cyclic goats in northern Mexico $\left(26^{\circ} \mathrm{N}\right)$ subjected to two administration schemes of prostaglandins (PG; 5 days vs. 10 days) to synchronize estrus response. Cyclic adult French-Alpine goats were allocated in two groups. Group $G 5(n=10)$, received the first PG-injection $(0.2 \mathrm{ml} ; 160 \mu \mathrm{g}$ cloprostenol) on $\mathrm{d}-5$ and group $\mathrm{G} 10(\mathrm{n}=10)$ received the first PG-injection on $d-10$. Both groups received the second PG-injection on $d 0$ (November 1). General reproductive outcomes after second PG-administration were similar $(P>0.05)$ between treatments. Estrus response= $60 \%$ for both groups, estrus latency $=68 \mathrm{~h}$ vs $52 \mathrm{~h}$, ovulation $=80 \%$ vs $60 \%$ (G5 vs G10, respectively; $P>0.05$ ). Since no differences occurred between groups, administration of the G5 treatment seems to be an interesting short-term synchronizing alternative protocol in that it generated important reproductive outcomes.

Keywords: French-Alpine goats; breeding season; estrus synchronization; prostaglandins; reproductive outcomes. 


\section{INTRODUCCIÓN}

En los pequeños rumiantes, la mayoría de los protocolos reproductivos para sincronizar los ciclos estrales durante la época de cría, se basan en el uso de tratamientos hormonales exógenos con diferentes dosis y regímenes de tiempo (Martemucci y D’Alessandro, 2011).

La prostaglandina $\mathrm{F}_{2 \alpha}$ es el principal agente luteolítico utilizado para sincronizar el estro durante la época reproductiva en estas especies, se metaboliza rápidamente por los pulmones, sin acumulación de tejido, siendo una alternativa interesante al uso de progestágenos, eCG y hCG (Omontese et al., 2016). No obstante, una desventaja del uso de prostaglandinas en comparación con eCG o hCG es que la ovulación está muy dispersa ya que la respuesta varía con la etapa del ciclo estral en el que se administran las prostaglandinas (Houghton et al. 1995). Después de la primera administración de prostaglandinas, ocurren ciclos de celo cortos y un porcentaje importante de hembras no responde a la segunda administración. De hecho, al reducir el período entre la primera y segunda administración de prostaglandinas de 10 a 7 días, se previene la ocurrencia de ciclos de celo cortos y se obtiene una mayor respuesta reproductiva (Maia et al., 2017). Se ha mencionado que, en cabras, el corpus luteum es sensible a PGF $_{2 \alpha}$ a partir del día 3 después del final del estro, obteniendo una alta proporción de hembras que presentan estro luego de la administración (Rubianes et al., 2003). Como se mencionó anteriormente, el uso de $\mathrm{PGF}_{2 \alpha}$ es un manejo reproductivo limpio ya que no deja residuos en los tejidos (Omontese et al., 2016) además, se puede incrementar el bienestar animal cuando se implementan estrategias reproductivas que hagan más rápido y eficiente el manejo del hato (Roger, 2012). Por lo tanto, los protocolos reproductivos nuevos e innovadores deben promover el bienestar animal, reduciendo el tiempo de manejo de los animales, reduciendo el uso de hormonas exógenas y disminuyendo los problemas de salud en el tracto reproductivo femenino. Dichas estrategias deberían disminuir, en paralelo, el costo de los tratamientos reproductivos así como el trabajo manual (Abecia et al., 2011; Gonzalez-Bulnes et al., 2011).

Basándonos en este fundamento, planteamos la hipótesis de que la administración de un protocolo de sincronización de PG a corto plazo (5 días) a las cabras durante la temporada de reproducción, debería producir resultados similares a los de los protocolos de administración de PG más largos (es decir, 10 o más días). Por lo tanto, el objetivo de esta investigación fue reducir el tiempo de tratamiento y evaluar la respuesta reproductiva de cabras cíclicas alpinas francesas en la Comarca Lagunera, México $\left(26^{\circ} \mathrm{N}\right)$. 


\section{MATERIALES Y MÉTODOS}

General

Todos los métodos y el manejo de las unidades experimentales utilizadas en este ensayo se realizaron en estricta conformidad con las pautas aceptadas para el uso ético, el cuidado y el bienestar de los animales en la investigación a nivel internacional (FASS, 2010), nacional (NAM, 2002) e institucional, con aprobación y número de referencia ITT513.2.2/1879/2014-5458-14P.

Ubicación, animales, manejo y grupos experimentales

El ensayo se llevó a cabo en el Instituto Tecnológico de Torreón (ITT), ubicado en el norte de México, en la Comarca Lagunera $\left(26^{\circ} 23^{\prime} \mathrm{N}, 104^{\circ} 47^{\prime} \mathrm{W}\right.$ y $\left.1.100 \mathrm{~m}\right)$; la duración del día es de $13 \mathrm{~h} 41 \mathrm{~min}$ en el solsticio de verano y de $10 \mathrm{~h} 19$ min en el solsticio de invierno. Se realizó de octubre a noviembre, correspondiente a la época de cría natural de la cabra. El estado de salud de todas las unidades experimentales fue controlado por un veterinario experimentado durante todo el período de prueba; no se produjeron problemas de salud durante el ensayo. Además, se hicieron esfuerzos para minimizar las posibles molestias en los animales de experimentación.

Las cabras franco-alpinas adultas cíclicas $(n=20 ; 3$ años de edad) se asignaron aleatoriamente a dos grupos homogéneos $(P>0,05)$ de acuerdo con el peso vivo $(P V)$ y la puntuación de la condición corporal (PCC: $1=$ emaciada y $4=$ grasa). El grupo G5 ( $n=10)$ tenía 45,9 $\pm 1,9 \mathrm{~kg}$ de peso corporal y 2,4 $\pm 0,2$ unidades de PCC; mientras que G10 $(n=10)$ y $46,8 \pm 1,7 \mathrm{~kg}$ PV y $2,5 \pm 0,1$ unidades PCC. Para sincronizar el estro, ambos grupos recibieron dos dosis intravulvar de un análogo de prostaglandina $(0,2 \mathrm{ml} ; 160 \mu \mathrm{g}$ de cloprostenol). Aunque los parámetros reproductivos de interés para el estudio están relacionados con la segunda inyección de PG, y el experimento fue diseñado para evaluar la respuesta reproductiva después de la segunda administración, administrada el mismo día (día 0; 1 de noviembre) para ambos grupos, los parámetros reproductivos se midieron después de la primera y segunda administración de PG. El día -5 (27 de octubre), G5 recibió una primera inyección de PG; mientras que G10 recibió la primera inyección de PG el día -10 (22 de octubre).

Todas las hembras fueron ubicadas en corrales abiertos de $6 \times 6 \mathrm{~m}$, separados $60 \mathrm{~m}$ entre sí. Las hembras tuvieron acceso ad libitum a agua potable y recibieron una dieta que cumplía con sus requerimientos nutricionales de mantenimiento, consistente en acceso ad libitum a heno de alfalfa (17\% de proteína cruda, PC; 1,9 MCal ME/kg DM) y $100 \mathrm{~g}$ de concentrado comercial (14\% PC, 2,7 MCal ME/kg DM), disponible durante todo el período experimental. En la Figura 1 se muestra una representación esquemática del procedimiento experimental. 
Evaluación de la actividad estral

Una vez que recibió la primera inyección de PG, se evaluó la actividad estral durante 20 días, dos veces al día (0800 y 1700 h) durante 15 minutos cada vez, utilizando un hombre sexualmente activo provisto de un delantal. Las hembras detectadas en celo de pie fueron apareadas al menos dos veces por un macho probado en fertilidad y libido.

Evaluación de la actividad ovárica y el embarazo.

Las cabras de ambos grupos se sometieron a escaneo ecográfico transrectal en tiempo real en modo B (matriz lineal Aloka SSD500; Overseas Monitor Corp.Ltd., Richmond, BC, Canadá) durante todo el período de investigación para detectar y medir las estructuras ováricas. El escaneo fue realizado por un operador experimental con una hembra en posición de pie. El transductor se insertó en el recto hasta que se observó una imagen de los ovarios en el monitor. Luego, se giró el transductor hasta que se escanearon ambos ovarios. Diariamente se midió el diámetro de los folículos (2-3, 4-5 y preovulatorio $\geq 6 \mathrm{~mm}$ ) y se registró su ubicación en los ovarios. El corpus luteum se identificó en escala de grises como un área hipoecoica dentro de un ovario. Después de ambas administraciones de PG, se detectó la ovulación midiendo el folículo preovulatorio con el diámetro máximo ( $\geq 6 \mathrm{~mm}$ ) y observando los cambios morfológicos dentro del ovario desde el tejido folicular hasta la formación de un corpus luteum. El diagnóstico de embarazo se realizó a los 45 días post-apareamiento mediante gammagrafía transrectal de acuerdo con los procedimientos descritos, con base en los hallazgos con la ecografía, se calcularon los porcentajes de ovulación y número de corpus luteum (ContrerasVillarreal et al., 2016; Medan et al., 2003).

\section{PROTOCOLO EXPERIMENTAL}

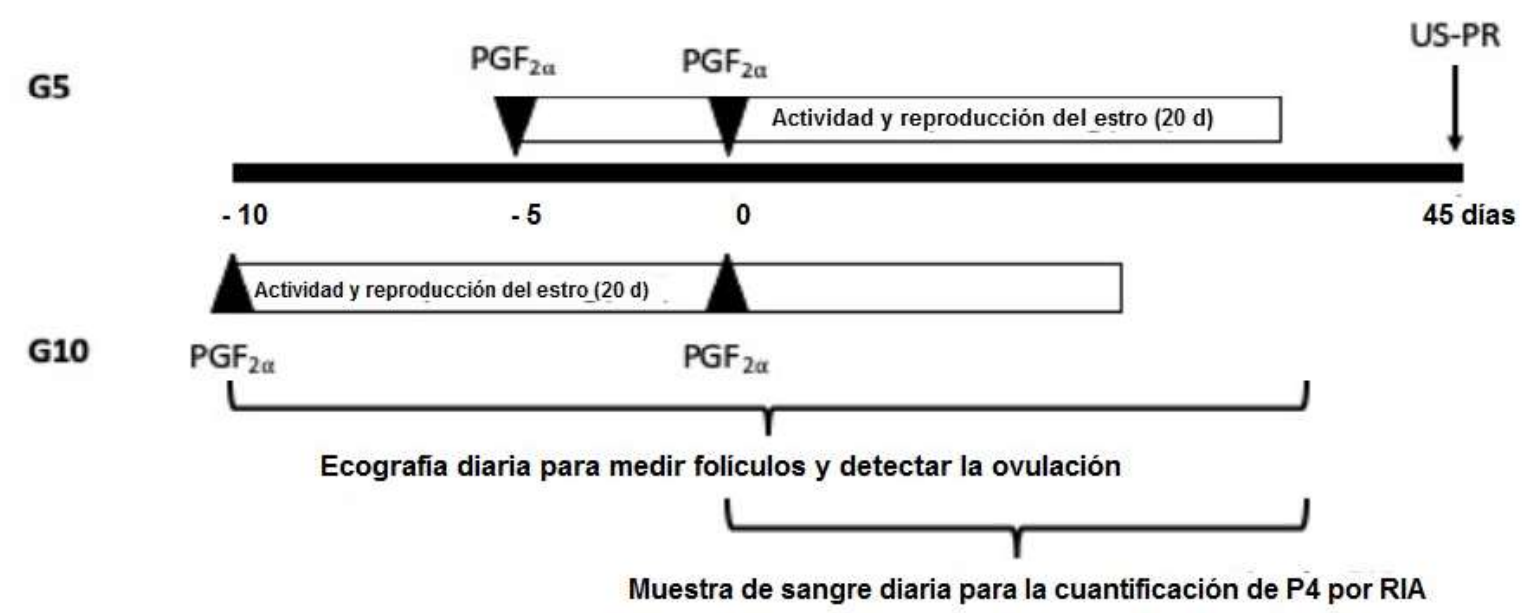

Figura 1. Una representación esquemática del protocolo experimental para sincronizar cabras lecheras cíclicas franco-alpinas adultas durante la temporada de reproducción (octubrenoviembre, $\left.\mathbf{2 6}^{\circ} \mathrm{N}\right)$ en el norte de México. Ambos grupos recibieron dos dosis de prostaglandina $(0,2 \mathrm{ml}$; $160 \mu \mathrm{g}$ de cloprostenol) para G5 el 27 de octubre y G10 el 22 de octubre. Luego, ambos grupos recibieron una segunda dosis el 1 de noviembre. 
Cuantificación de las concentraciones plasmáticas de progesterona Se realizó una toma de sangre diaria mediante punción venosa yugular a partir de la segunda administración de PG y con una duración de 10 días. La sangre se centrifugó y el plasma se recogió por duplicado y se almacenó a $-20^{\circ} \mathrm{C}$ hasta el análisis hormonal. La concentración plasmática de $\mathrm{P} 4$ se determinó mediante radioinmunoanálisis (RIA), utilizando un kit RIA comercial (Diagnostic Products, Los Ángeles, CA, EE. UU.) Validado para plasma de rumiantes (Schneider y Hallford, 1996). Los coeficientes de variación intra e interensayo fueron 9,9 y 12,3\%, respectivamente. Mientras que la recuperación media fue del $94 \%$, la sensibilidad del ensayo fue de $0,1 \mathrm{ng} / \mathrm{ml}$.

\section{Análisis estadístico}

Los datos sobre los porcentajes de cabras en estro y preñadas se analizaron mediante procedimientos categóricos utilizando el procedimiento GENMOD de SAS (SAS Inst. Inc., Cary, NC, EE UU.), con la función de enlace logit. El único efecto incluido en el modelo fue el tratamiento, con cada animal considerado como una sola unidad experimental. Se utilizó un análisis de varianza unidireccional (PROC GLM) para un diseño completamente aleatorizado para probar los efectos de los tratamientos sobre la aparición del estro, la ovulación, el inicio del estro y la progesterona. Según el diseño experimental, solo se analizaron los niveles plasmáticos de $\mathrm{P} 4$ para ambos grupos del $\mathrm{d} 0$ al $\mathrm{d} 10$. Las diferencias estadísticas entre tratamientos se consideraron significativas a $\mathrm{P}<0.05$.

\section{RESULTADOS Y DISCUSIÓN}

Los resultados muestran que los tratamientos G5 y G10 generan respuestas reproductivas similares $(P>0.05)$ en cabras cíclicas alpinas francesas durante la temporada de reproducción. No obstante, después de la segunda inyección de PG, hubo una tendencia $(P=0,06)$ a obtener una mayor tasa de embarazo en las mujeres del grupo G5. En la Tabla 1 se presenta un resumen de estos datos.

Las concentraciones de progesterona plasmática después de la segunda administración de PG para ambos grupos experimentales se muestran en la Figura 2. Las hembras de ambos grupos respondieron a la inyección de $P G$, registrando una disminución dramática en las concentraciones de $P 4$, sin diferencias significativas $(P>0.05)$ entre grupos experimentales del d0 al d9 de la investigación. 
Tabla 1. Respuesta reproductiva de cabras alpinas francesas sometidas a dos administraciones de prostaglandinas ( $160 \mu \mathrm{g}$ de Cloprostenol cada una), con un intervalo de administración de 5 (G5) y 10 (G10) días durante la temporada reproductiva

\begin{tabular}{|c|c|c|c|c|c|c|}
\hline & \multicolumn{3}{|c|}{$1^{\circ}$ inyección $P G$} & \multicolumn{3}{|c|}{$2^{\circ}$ inyección PG } \\
\hline & G5 & G10 & Valor $p$ & G5 & G10 & Valor $p$ \\
\hline Estro (\%) & $80(8 / 10)$ & $90(9 / 10)$ & .56 & $60(6 / 10)$ & $60(6 / 10)$ & 1 \\
\hline $\begin{array}{l}\text { Latencia del estro } \\
\text { (h) }\end{array}$ & $48 \pm 0.0$ & $79 \pm 16.9$ & .09 & $68 \pm 13.4$ & $52 \pm 2.5$ & .26 \\
\hline Ovulación (\%) & $100(10 / 10)$ & $100(10 / 10)$ & & $80(8 / 10)$ & $60(6 / 10)$ & .36 \\
\hline $\begin{array}{l}\text { Tasa de embarazo } \\
(\%) \\
\text { Folículos }\end{array}$ & ------- & ------- & ----- & $60(6 / 10)$ & $20(2 / 10)$ & .06 \\
\hline $\begin{array}{l}\text { ovulatorios (mm y } \\
\mathrm{n} \text { ) }\end{array}$ & $8.9 \pm 0.3(10)$ & $9.7 \pm 0.6(10)$ & .32 & $9.5 \pm 0.4(8)$ & $9.3 \pm 0.3(6)$ & .96 \\
\hline $\begin{array}{l}\text { Promedio del } \\
\text { Corpus Luteum (n) }\end{array}$ & $1.1 \pm 0.1(9)$ & $1.0 \pm 0.0(6)$ & .4 & $1.0 \pm 0.0(8)$ & $1.3 \pm 0.3(6)$ & .29 \\
\hline
\end{tabular}

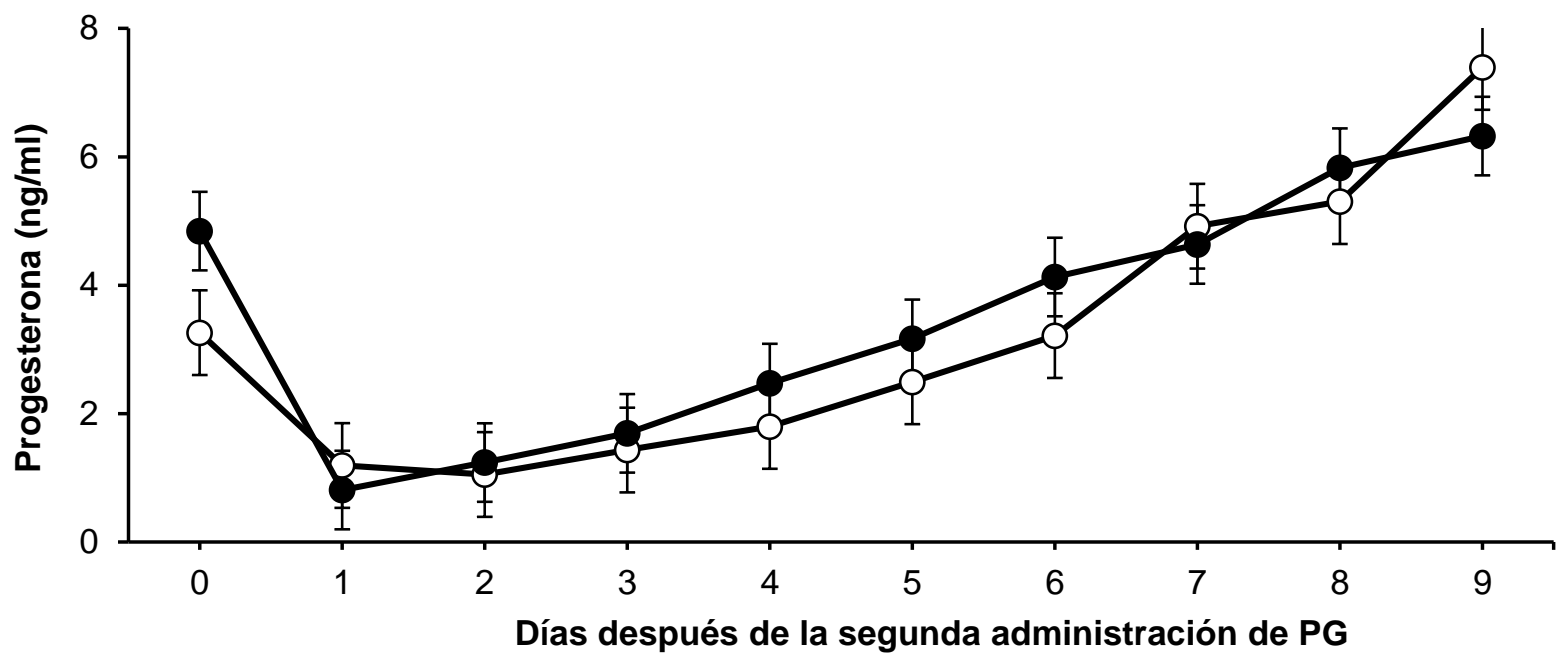

Figura 2. Niveles plasmáticos de progesterona para los grupos G5 (círculos blancos) y G10 (círculos negros) después de la segunda administración de prostaglandinas (d0; $\mathrm{P}>0.05$ )

La respuesta de estro observada después de la segunda inyección de PG en el G5 fue similar a las reportadas en estudios previos realizados a intervalos de 9 a 11 días, con respuestas de estro de 70\% a 85\% (Omontese et al., 2016; Freitas et al., 2004). Además, el $80 \%$ de las hembras ovularon después de la segunda inyección de PG, sin diferencias con respecto al grupo G10 ni en el porcentaje de ovulación ni en el tamaño y número de folículos ovulatorios [ 80 vs $60 \%$ y $9,5 \pm 0,4$ (8) vs $9,3 \pm 0,3$ (6), respectivamente; $P>0,05$ ]. 
Estos resultados son similares a otros estudios en los que se aplicaron dos inyecciones de PG en intervalos de 10 a 12 días (Al Yacoub et al., 2011; Kusina et al., 2001). Nuestros hallazgos también están en línea con Martemucci y D'Alessandro (2011), quienes usaron progestágenos, prostaglandinas y eCG durante 5 días con el $80 \%$ de la actividad ovulatoria.

El grupo G5 mostró una tasa de embarazo del 60\% y una fertilidad del 100\% (6/6), lo que sugiere que respondieron a dos administraciones de PG con un intervalo de cinco días, que es un intervalo más corto que el utilizado por Al Yacoub et al. (2011), quienes aplicaron dos administraciones de cloprostenol entre los 6 y 13 días del ciclo estral, y lograron la misma respuesta reproductiva con un protocolo de inseminación de tiempo fijo. Otro aspecto importante a destacar de nuestro protocolo es que el nivel de PG utilizado ( $160 \mu \mathrm{g}$ de cloprostenol) es inferior a la mayoría de los niveles utilizados en otros estudios (250 $\mathrm{\mu g}$ de cloprostenol), reduciendo la dosis en torno al 40\% (Omontese et al., 2016).

Con respecto a los resultados reproductivos observados de G10, el porcentaje de mujeres que respondieron a la segunda inyección de PG puede considerarse bajo, ya que el $20 \%$ tuvo un ciclo estral corto (5,5 días) antes de la segunda inyección de PG, con un nivel de P4 plasmático más alto de $1 \mathrm{ng} / \mathrm{ml}$ (Titi et al., 2010). Dicho porcentaje de ciclos estrales cortos es similar a los obtenidos en otros estudios que utilizan cloprostenol como agente sincronizador, lo que sugiere que este análogo de PG puede promover un crecimiento y una funcionalidad deficientes del tejido lúteo (Vázquez et al., 2010). Asimismo, esto podría explicarse por la gran variabilidad encontrada cuando se administran prostaglandinas (Houghton et al. 1995), dado que hubo una mayor ventana de tiempo desde la primera administración a la segunda, los animales se encontraban en diferentes estadios foliculares y esto se reflejó en una menor tasa de embarazo, ya que las ovulaciones no estaban sincronizadas. Además, el $20 \%$ restante de las mujeres que no respondieron a la segunda inyección de PG sugiere que el corpus luteum existente no se suprimió inmediatamente después de la primera aplicación, un escenario respaldado por las concentraciones plasmáticas de P4 observadas (Figura 2). Además, solo el $60 \%$ de las ovulaciones ocurrieron en $\mathrm{G} 10$ después de la segunda inyección de PG, un resultado que puede considerarse bajo con respecto a otros estudios que alcanzaron el $90 \%$ al 100\% (Al Yacoub et al., 2011; Kusina et al., 2001).

Además, la tasa de embarazo y la fertilidad de las hembras del G10 fue menor de lo esperado (20\% y $33 \%$, respectivamente). De hecho, del $60 \%$ de las hembras que presentaron celo permanente y ovulación después de la segunda administración de PG, cuatro de ellas repitieron el celo entre 5 y 12 días después de la reproducción. Tal respuesta sugiere que el proceso de luteogénesis generado después del tratamiento con $P G$, provocado por folículos de baja calidad, no pudo mantener una síntesis de P4 
adecuada para sostener el embarazo (Al Yacoub et al., 2011). En las cabras, como en otros mamíferos, los primeros días después de la ovulación son fundamentales para potenciar la luteogénesis, así como para promover la implantación embrionaria, la embriogénesis y el reconocimiento materno de los procesos de gestación (Vázquez et al., 2010).

Con base en los resultados reproductivos descritos por las hembras G5, nuestro estudio genera información sobre la reducción del tiempo requerido para promover la aparición del estro con el uso de hormonas exógenas para el manejo reproductivo de pequeños rumiantes. Además, también es posible reducir el riesgo sanitario en el tracto reproductivo y al mismo tiempo esperar una disminución en el costo de los tratamientos hormonales al reducir la dosis requerida (Omontese et al., 2016; Abecia et al., 2011). Estos resultados adquieren especial importancia si se considera que, en la mayoría de los protocolos de sincronización en cabras y ovinos, el uso de prostaglandinas considera un conocimiento preciso de la fase del ciclo estral, (es decir, la fase lútea), aplicando una mayor cantidad de prostaglandinas solo o en combinación con progestágenos u otras hormonas.

\section{CONCLUSIÓN}

La administración de un protocolo basado en prostaglandinas a corto plazo en un intervalo de cinco días para sincronizar la actividad del estro de las cabras cíclicas alpinas francesas durante la temporada de reproducción, generó resultados reproductivos importantes al considerar la actividad del estro, la latencia del estro, la función lútea y los niveles plasmáticos de progesterona, así como tasas de ovulación y preñez, junto con la reducción del número de días manejando las cabras; siendo el último de importancia fisiológica y de importancia reproductiva para la industria caprina.

Agradecimientos: Reconocemos el apoyo financiero brindado por el fondo sectorial del Consejo Nacional de Ciencia y Tecnología (CONACyT, México) y la Secretaría de Agricultura, Ganadería, Desarrollo Rural, Pesca y Alimentación (SAGARPA, México): 2017-4-291691.

\section{LITERATURA CITADA}

ABECIA JA, Forcada F, Gonzalez-Bulnes A. 2011. Pharmaceutical control of reproduction in sheep and goats. Veterinary Clinic of North America Food Animal Practice. 27: 67-79. https://doi.org/10.1016/j.cvfa.2010.10.001

AL YACOUB AN, Gauly M, Sohnrey B, Holtz W. 2011. Fixed-time deep uterine insemination in $\mathrm{PGF}_{2 \alpha}$-synchronized goats. Theriogenology. 76: 1730-1735. https://doi.org/10.1016/j.theriogenology.2011.07.005 
CONTRERAS-VILLARREAL V, Meza-Herrera CA, Rivas-Muñoz R, Angel-Garcia $O$, Luna-Orozco JR, Carrillo E, Mellado M, Véliz-Deras FG. 2016. Reproductive performancee of seasonally anovular mixed-bred dairy goats induced to ovulate with a combination of progesterone and eCG or estradiol. Animal Science Journal. 87(6):7505. https://doi.org/10.1111/asj.12493

FASS, 2010. Guide for the care and use of agricultural animals in agricultural research and teaching. 3rd Edition, FASS, Champaing, IL, USA. Pp. 177. ISBN: 978-1-7362930$0-3$

FREITAS JVF, Rondina D, Lopes Junior ES, Teixeira DIA, Paula NRO. 2004. Hormonal treatments for the synchronization of oestrus in dairy goats raised in the tropics. Reproduction Fertility and Development. 16: 415-420. https://doi.org/10.1071/RD04031

GONZALEZ-BULNES A, Meza-Herrera CA, Rekik M, BenSalem H, Kridli RT. 2011. Limiting factors and strategies for improving reproductive outputs of small ruminants reared in semi-arid environments. In: Degenovine, K.M. (Ed.), Emi-arid Environments: Agriculture, Water Supply and Vegetation. Nova Science Publishers Inc., Hauppauge, NY, USA. Pp. 41-60 (Chapter 2) ISBN: 978-1-61761-541-2.

HOUGHTON JAS, Liberati N, Schrick FN, Townsend EC, Dailey RA, Inskeep EK. 1995. Day of estrus cycle affects follicular dynamics after induced luteolysis in ewes. Journal of Animal Science. 73(7):2094-2101. https://doi.org/10.2527/1995.7372094x

KUSINA NT, Chinuwo T, Hamudikuwanda H, Ndlovu LR, Muzanenhamo S. 2001. Effect of different dietary energy level intakes on efficiency of estrus synchronization and fertility in Mashona goat does. Small Ruminant Research. 39: 283-288. https://doi.org/10.1016/S0921-4488(00)00192-9

MAIA ALRS, Brandao FZ, Souza-Fabjan JMG, Balaro MFA, Oliveira MEF, Facó O, Fonseca JF. 2017. Reproductive parameters of dairy goats after reveiving two doses of d-cloprostenol at different intervals. Animal Reproduction Science. 181: 16-23. https://doi.org/10.1016/j.anireprosci.2017.02.013

MARTEMUCCI G, D'Alessandro AG. 2011. Induction-synchronization of oestrus and ovulation in dairy goats with different short-term treatments and fixed time intrauterine or exocervical insemination system. Animal Reproduction Science. 126: 187-194. https://doi.org/10.1016/j.anireprosci.2011.05.011

MEDAN MS, Watanabe G, Sasaki K, Sharawy S, Groome NP, Taya K. 2003. Ovarian dynamics and their associations with peripheral concentrations of gonadotropins, ovarian steroids, and inhibin during the estrous cycle in goats. Biology of Reproduction. 69: 5763. https://doi.org/10.1095/biolreprod.102.013334 
National Academy of Medicine. 2002. Guide for the care and use of laboratory animals. Co-produced by the National Academy of Medicine-Mexico and the Association for assessment and accreditation of laboratory animal care international. 1st. Edition, Harlan Mexico, DF, Mexico. ISBN-13: 978-0-309-15400-0

OMONTESE BO, Rekwot PI, Ate IU, Ayo JO, Kawa MU, Rwuaan JS, Nwannenna Al, Mustapha RA, Bello AA. 2016. An update on oestrus synchronization of goats in Nigeria. Asian Pacific Journal of Reproduction. 5: 96-101. https://doi.org/10.1016/j.apjr.2016.01.002

ROGER, PA. 2012. Welfare issues in the reproductive management of small ruminants. Animal Reproduction Science. 130(3-4):141-146.

https://doi.org/10.1016/j.anireprosci.2012.01.007

ROMANO JE, Alkar A, Amstalden M. 2016. Effect of copulation on estrus duration and ovulation time in goats. Theriogenology. 85(2):330-334. https://doi.org/10.1016/j.theriogenology.2015.09.021

RUBIANES E, Menchaca A, Carbajal B. 2003. Response of the 1-5 day-aged ovine corpus luteum to prostaglandin F2a. Animal Reproduction Science. 78(1-2):47-55. https://doi.org/10.1016/S0378-4320(03)00046-0

SCHNEIDER RJ, Hallford DM. 1996. Use of a rapid progesterone radioimmunoassy to predict pregnancy and fetal numbers in ewes. Sheep and Goat Research Journal. 12: 3338. https://agris.fao.org/agris-search/search.do? recordID=US1997067677

TITI HH, Kridli RT, Alnimer MA. 2010. Estrus synchronization in sheep and goats using combinations of $\mathrm{GnRH}$, progestagen and prostaglandin $\mathrm{F} 2 \alpha$. Reproduction in Domestic Animals. 45: 594-599. https://doi.org/10.1111/j.1439-0531.2008.01309.x

VÁZQUEZ MI, Blanch MS, Alanis GA, Chaves MA, Gonzalez-Bulnes A. 2010. Effect of treatments with a prostaglandin analogue on developmental dynamics and functionality of induced corpora lutea in goats. Animal Reproduction Science. 118: 42-47. https://doi.org/10.1016/j.anireprosci.2009.05.016 